\title{
FORTALECER A LOS GOBIERNOS MUNICIPALES EN MÉXICO A TRAVÉS DE LA ADMINISTRACIÓN ESTRATÉGICA
}

Manuel Zavaleta Suárez ${ }^{1}$

RESUMEN. La importancia que representa el municipio en el sistema político nacional se debe a que éste es el orden de gobierno más cercano a la población, y el cual debería ser el proveedor más eficiente de servicios públicos, además de que es aquí donde convergen inicialmente la mayoría de los procesos sociales, estas razones son las que han llevado a exaltar la necesidad de dotar al municipio de las facultades legales, de los elementos administrativos, técnicos y financieros, para que este orden de gobierno pueda brindar los servicios y satisfacción de las demandas sociales.

Palabras claves: Municipio, Desarrollo, Fortalecimiento, Estrategia, RIG's

Abstract.The importance that represents the city in the national political system is because this is the order of closest to the town government, and which should be the most efficient provider of public services, and that is where initially converge most of social processes, these are the reasons that have led to extol the need to give the municipality the legal authority, administrative, technical and financial elements, so that this order of government can deliver the services and satisfaction of social demands.

Keywords: City, Development, Strengthening, Strategy, RIG's

\footnotetext{
${ }^{1}$ Doctor en Ciencias Administrativas, por la UABC; con Maestría en Administración Pública, por la Facultad de Ciencias Sociales y Políticas de la UABC; es Licenciado en Política y Gestión Social por la Universidad Autónoma Metropolitana unidad Xochimilco UAM-X, cuenta con el reconocimiento al Perfil Deseable del Programa para el Desarrollo Profesional Docente (PRODEP de la SEP). Actualmente es coordinador de posgrado e Investigación en la FCSyP UABC, donde se desempeña como profesor de tiempo completo en diferentes áreas de especialidad de las licenciaturas en Administración Pública, Relaciones Internacionales y Economía. Asimismo, ha impartido clases a nivel Posgrado, y en la Facultad de Derecho de la UABC. Como servidor público ha colaborado en la Dirección de Vivienda y Reservas Territoriales de la Secretaría de Infraestructura y Desarrollo Urbano del Gobierno del Estado de Baja California, donde ha desarrollado diferentes proyectos de investigación, todos encaminados al fomento del Desarrollo Urbano, la promoción de Vivienda y Reservas Territoriales.
} 


\section{Introducción}

El sistema político mexicano es producto de una tradición altamente centralista, la cual ha generado grandes desequilibrios entre las regiones que conforman nuestro país y una débil eficacia en las políticas y programas gubernamentales, lo que sin duda ha constituido un obstáculo para la modernización y el desarrollo del México (Cabrero, 2003).

En este escenario centralista en el que ha estado inmerso nuestro país, el gobierno federal siempre se ha colocado por encima de la soberanía de los gobiernos estatales y municipales, los cuales al no contar con los recursos necesarios, con las atribuciones requeridas, y con la libertad de decisión necesarias, afrontan dificultades para ejercer con eficiencia sus funciones de gobierno (Cabrero, 2004).

La fragilidad que han mantenido los gobiernos locales puede ser atribuida a una multiplicidad de factores relacionados con la ausencia de una visión de largo plazo, la escases de recursos, ausencia de cooperación en las relaciones intergubernamentales, falta de profesionalización de los recursos humanos y la poca institucionalización social, así como a la ausencia de un sistema de planeación y de instrumentos de gestión que propicien el desarrollo local (Ziccardi, 2002; Guillén, 2003; Cabrero, 2004).

La importancia que representa el municipio en el sistema político nacional se debe a que éste es el orden de gobierno más cercano a la población, y el cual debería ser el proveedor más eficiente de servicios públicos, además de que es aquí donde convergen inicialmente la mayoría de los procesos sociales, estas razones son las que han llevado a exaltar la necesidad de dotar al municipio de las facultades legales, de los elementos administrativos, técnicos y financieros, para que este orden de gobierno pueda brindar los servicios y satisfacción de las demandas sociales (Peters, 2005 y Navas, 2010).

Los primeros indicios por resaltar la importancia que deberían tener los gobiernos locales se dan con dos reformas al artículo 115 constitucional realizadas entre 1983 y 1999. En este 
sentido, la presente investigación coincide con la idea de Guillen (2001) cuando señala que la reforma municipal no puede concebirse a partir de un modelo único para todo el país, promover el desarrollo de esta instancia de gobierno significa resaltar su particularidad y sus necesidades específicas, así como la organización de sus asuntos públicos y de gobierno.

Es así que a partir de las últimas cuatro décadas el gobierno municipal ha comenzado a tener mayor relevancia en la vida nacional; muestra de ello es el surgimiento de una serie de proyectos enfocados en propiciar que los gobiernos municipales alcancen un desarrollo integral y que éstos a su vez contagien la transformación del sistema político en su conjunto.

Un ejemplo de este tipo de proyectos es el que desarrolla la Organización de las Naciones Unidas (ONU), la cual en 1993 da a conocer en el marco de la cumbre de Rio, la Agenda Local XXI, que representa un proceso para la definición de políticas locales para el desarrollo sustentable; con este proyecto se trata de atender diferentes planes de acción enfocados en alcanzar el desarrollo sustentable en el siglo XXI.

Ese esfuerzo a nivel internacional sin lugar a duda influyó para que el Gobierno de nuestro país enmarcara la importancia que tienen actualmente los gobiernos municipales, por lo cual en el Plan Nacional de Desarrollo (2007-2012) establece que el gobierno local se convertirá en actor fundamental del pacto federal y éste tendrá una mayor participación en la planeación y programación de las políticas públicas sectoriales y regionales, como corresponde al sentido del artículo 115 de la constitución mexicana sobre el fortalecimiento del municipio libre.

Para alcanzar este propósito se ha puesto en marcha el programa especial Hacia un Auténtico Federalismo en el cual se encuentran identificadas una serie de directrices para promover una nueva faceta del federalismo mexicano e impulsar el desarrollo de los gobiernos municipales. 
Una de esas estrategias es el programa Agenda para el Desarrollo Municipal el cual es promovido por la Secretaría de Gobernación a través del Instituto Nacional para el Federalismo y Desarrollo Municipal (INAFED, 2012).

El programa trata de brindar una solución factible al problema existente de la subordinación que prevalece en los gobiernos municipales. Su objetivo es: “aportar los elementos necesarios para que los gobiernos locales alcancen las condiciones mínimas, en los distintos rubros de políticas y servicios públicos que les permitan asumir y ejercer las responsabilidades y recursos transferidos por los otros órdenes de gobierno de forma más eficiente y transparente" (INAFED, 2012).

La importancia de Agenda para el Desarrollo Municipal se debe a que permite desarrollar un proceso de Administración estratégica que resulta de gran importancia para la vida municipal; este proceso surge mediante el empleo de una metodología que permite identificar a través de un autodiagnóstico la situación que guarda la administración local y partir de ahí generar políticas públicas que permitan potenciar las fortalezas y mitigar las debilidades localizadas.

A pesar de que la idea de fomentar el desarrollo de los gobiernos locales se ha convertido en una prioridad para el país, se puede observar que aún la participación de éstos en la vida nacional es muy limitada ya que su actuación se reduce sólo a la prestación limitada de algunos servicios públicos lo cual queda muy al margen de los grandes proyectos impulsados por el Gobierno Federal (Sánchez y García, 2007).

Resulta indispensable construir un modelo de gestión en el ámbito local que a través de un proceso de administración estratégica favorezca el fortalecimiento del ámbito de gobierno.

La creación del modelo tendrá éxito siempre y cuando el proceso contemple los siguientes factores: 
- La generación de un diagnóstico sistemático como el que propone Agenda para el Desarrollo Municipal y en el cual se identifiquen tanto las fortalezas como las debilidades con las que trabaja actualmente la administración municipal.

- Una vez identificadas las áreas de oportunidad, partir de ahí para generar un proceso de políticas públicas en las que se formulen y se pongan en marcha una serie de estrategias que potencialicen las fortalezas y erradiquen las debilidades localizadas.

- Será necesario una redistribución de funciones y competencias, es decir, una modificación de las relaciones intergubernamentales que se desarrollan actualmente, en las que se promueva la cultura de la gestión compartida, intercomunicada y corresponsable a la conducción de las estrategias y líneas de acción del gobierno municipal.

El presente proyecto se orienta hacia la búsqueda de factores determinantes respecto a la incorporación de un Modelo de Gestión en beneficio de la administración municipal. Por lo cual, el sustento teórico de este proyecto estará enfocado en los aspectos relacionados con fortalecimiento municipal, con las políticas públicas, con las relaciones intergubernamentales (RIG`s) y con la administración estratégica.

\section{Fortalecimiento Municipal}

La excesiva centralización, administrativa, económica y política del gobierno federal ha sido la constante que ha dominado el sistema político mexicano durante todo el siglo XX, lo que ha dado como resultado que el gobierno federal se coloque por encima de la soberanía de los estados y los municipios. Este hecho hizo que en México los gobiernos locales afrontaran frecuentemente dificultades para ejercer con eficiencia sus funciones de gobierno, por lo cual, éstos no han podido ejercer la relevancia que la Constitución les otorga. 
Esto ha generado que durante el transcurso de todo el siglo $\mathrm{XX}$, se hayan visto gobiernos municipales con poco o nula autonomía y por ende, dificultades para que ellos mismos ejerzan sus propias decisiones.

Ante tal situación, el anhelo por fortalecer al gobierno municipal se convirtió en una constante en el discurso político de México, donde se exaltaba que habría que generar estrategias para "dotar al municipio de las facultades legales, de los elementos jurídicos, administrativos, técnicos y financieros, para que pueda abordar los servicios públicos ${ }^{2}$ mínimos a que todo municipio está obligado" (INAP; 1995: 46).

La idea de fortalecer a los gobiernos locales tomó consistencia en 1983 con la reforma al artículo 115 constitucional; en la que se identificaban por primera vez cuáles serían los ingresos que pertenecen al municipio; así como una serie de facultades que la ley le otorga para administrarlos; al mismo tiempo se definían cuáles serían los servicios públicos que le competen; además, se establecían las bases de su autonomía para reglamentar la vida municipal; con esta reforma se dotó al municipio de: recursos, funciones, patrimonio propio y personalidad jurídica para actuar (Guzmán; 1995). Se pretendía promover una descentralización de la vida municipal en aras de generar territorios competitivos, mediante el aprovechamiento eficiente de los recursos endógenos, la creación de entornos locales innovadores y la cooperación estratégica de diferentes actores (Vásquez, 2000; Silva, 2003 y Alburquerque, 2001).

Como bien señala Guzmán (1995), esta reforma se hizo pensando en un municipio urbano, “poniendo la imaginación en el municipio ideal, con un ayuntamiento eficaz que cobra los

\footnotetext{
${ }^{2}$ Se reconocen los siguientes: a) agua potable y alcantarillado, b) alumbrado público, c) limpia, d) mercados y centrales de abasto, e) panteones, t) rastro, g) calles, parques y jardines, h) seguridad pública y tránsito, i) Los demás que las legislaturas locales determinen según las condiciones territoriales y socioeconómicas de los Municipios, así como su capacidad administrativa y financiera. Art. 115, Constitución Política de los Estados Unidos Mexicanos.
} 
impuestos, obtiene recursos de los servicios públicos que presta y gasta su dinero en equipamientos, etc., sin considerar el otro extremo, el del municipio rural y paupérrimo que sobrevive apenas de las participaciones fiscales otorgadas, pero que no prestan ningún servicio".

En este sentido Guillen (2001) señala que "La reforma municipal no puede concebirse a partir de un modelo único para todo el país, [...] fortalecer a esta instancia de gobierno significa resaltar su particularidad y sus necesidades específicas de desarrollo, así como la organización de sus asuntos públicos y de gobierno".

Este primer intento por brindarle mayores atribuciones al municipio tomó otro rumbo con la llegada de Carlos Salinas de Gortari a la presidencia (1988 - 1994), aunque al igual que su predecesor se comprometió a concentrar sus esfuerzos por impulsar la descentralización y en continuar los esfuerzos por afianzar la reforma municipal.

Dichos compromisos no dieron muchos frutos ya que con el propósito de frenar el avance de la oposición e impulsar su proyecto de reforma económica, el gobierno federal centralizó nuevamente el poder, por lo cual el proceso de descentralización no figuró como un elemento fundamental de su agenda política, su presencia estaba implícita dentro del Programa Nacional de Solidaridad, el cual tenía como propósito beneficiar a la población con mayor índice de pobreza a través de subsidios directos en infraestructura, vivienda y otros servicios sociales.

Con la llegada de Ernesto Zedillo a la presidencia (1994-2000) también se da una severa crisis económica, la cual agudiza los defectos del sistema económico y político centralista que el gobierno venía aplicando durante mucho tiempo en México. Esta crisis dio la pauta para que varios gobernadores y presidentes municipales presionaran al gobierno federal para obtener un mayor poder de decisión en la construcción de alternativas de política pública a través de foros y mecanismos de consulta. 
Uno de los principales foros tuvo lugar en Guadalajara en marzo de 1995 denominado Foro nacional Hacia un Auténtico Federalismo, sirvió para que diferentes actores exaltaran sus demandas hacia el gobierno federal; reprochándole que es imprescindible la necesidad de adelgazar al gobierno central mediante la reasignación de facultades, atribuciones y recursos económicos a estados y municipios.

Al mismo tiempo dicho foro sirvió como vitrina para que Ernesto Zedillo difundiera su nueva política de descentralización que denominó Nuevo Federalismo; dicho programa pretendía forjar las bases para el fortalecimiento tanto de la democracia como del gobierno local, así como consolidar la unidad nacional y propiciar un México más equilibrado y justo. El objetivo que regía la actuación de dicho programa era "fortalecer la unidad nacional a partir de reivindicar la función compensatoria del Pacto Federal; vigorizar la autonomía política de los estados con base en relaciones intergubernamentales sustentadas en los principios de cooperación y coordinación y mediante una profunda redistribución de facultades, funciones, responsabilidades y recursos del Gobierno Federal hacia los gobiernos estatales y el Municipio" (Programa para un Nuevo Federalismo).

Otro de los logros que se dieron en esta administración fue la reforma constitucional realizada en 1999 al artículo $115^{3}$. Algunos de los avances en materia de fortalecimiento municipal se pueden sintetizar de la siguiente manera:

Se define con mayor claridad el carácter de la autoridad municipal, sus competencias propias, sus alcances y los límites con respecto al poder estatal. Además otorga al municipio el carácter de órgano de gobierno, dotado de competencias exclusivas, que sólo el ayuntamiento

\footnotetext{
${ }^{3}$ Para profundizar más sobre el tema de las reformas que ha sufrido el artículo 115 constitucional véase Robles Martínez Reynaldo, El Municipio, Miguel Ángel Porrúa, México, 2003
} 
podrá transferir a los estados, se considera que requiere de su asistencia para cubrirlas. Esto fortalece al municipio frente a la entidad y dificulta la formación de organismos paralelos al ayuntamiento.

Al conservar el presidente municipal el mando de las políticas preventivas se garantiza el derecho de iniciativa en materia tributaria y se fortalece el carácter fiscalizador de las legislaturas en las propias finanzas. Mediante esta mejora el gobierno municipal podrá expedir normas sobre su organización, funcionamiento, administración interna y decidirá sobre la prestación y destino de los servicios públicos, además, para asegurar la eficiencia en la prestación de estos servicios, podrá asociarse libremente con otros municipios siempre y cuando se cuente con la autorización de los congresos locales.

Al concluir la administración de Ernesto Zedillo, también se dio por terminada la hegemonía que hasta entonces había tenido el Partido Revolucionario Institucional (PRI); este hecho trajo consigo aires de renovación en el sistema político mexicano; ejemplo de ello se puede apreciar en el Plan Nacional de Desarrollo 2000 - 2006 en el que se establecía la necesidad de avanzar hacia un auténtico federalismo, que permita lograr un mayor equilibrio en las relaciones intergubernamentales; precisar y distribuir el ejercicio de la función pública entre los órdenes de gobierno, fortalecer las haciendas públicas locales, favorecer la rendición de cuentas y motivar nuevos espacios de participación ciudadana, a fin de generar condiciones más favorables para el desarrollo de las comunidades.

Uvalle (1997) señala que para pensar en un federalismo auténtico hay que hablar de descentralización, la cual se encuentra inmersa en el federalismo trataría de conformar distintos centros neurálgicos de toma de decisiones periféricos con autonomía relativa respecto al núcleo central (Mejía, 2003); esto significa que los gobiernos estatales y municipales, que poseen autonomía relativa, se conformen como puntos importantes para la toma de decisiones consensuales, y que en forma conjunta con el gobierno federal favorezcan 
la coordinación y colaboración de la gestión pública para que esta se traduzca en respuestas efectivas para los ciudadanos. Un modo adecuado para lograr el enlace entre estas instancias de gobierno es mediante las relaciones intergubernamentales.

La política de descentralización favorece el desarrollo local a través de instituciones políticas distintas a las centrales, con formas de organización social propias de un régimen democrático, es modelo para sociedades diversas, faltas de organización y autonomía de los grupos sociales y políticos.

En este sentido el binomio "Descentralización y Federalismo es hacer coincidir diversas necesidades: que funcionen mejor nuestras instituciones políticas (más democracia); que funcionen mejor las instancias administrativas (más eficacia); que se fortalezca la organización territorial del Estado y que se amplíen cauces a las relaciones entre el Estado y la sociedad" (Moreno, 1998).

\section{El rol del federalismo en México}

El federalismo ${ }^{4}$ es una doctrina política que aporta conceptos sobre la forma en que deben organizarse las instituciones gubernamentales en un Estado nacional, ya que "es un creador y un reproductor de instituciones que dan sustento y operación al ejercicio del poder" (Uvalle, 2001).

Así, la organización del poder en un sistema federal es una dualidad, ya que en él coexisten un gobierno general (denominado nacional o federal) y, por el otro, un indeterminado número de gobiernos locales con determinadas facultades políticas, sin que ello signifique

\footnotetext{
${ }^{4}$ El origen del término Federalismo proviene del latín foedus, que significa alianza, pacto, acuerdo. En este sentido, el término federalismo hace referencia al "arreglo político institucional basado en una distribución funcional y territorial del poder entre un ámbito central y ámbitos locales (estados, provincias o territorios) independientes o federados, los cuales participan de un pacto que se sustenta en la Constitución (Baca, 2000: 238).
} 
subordinación o dominación entre ellos o entre el gobierno nacional y los gobiernos subnacionales (Guillén, 2001).

Para tratar de fomentar un auténtico pacto federal en el plan nacional de desarrollo de la administración de Vicente Fox (2000-2006) se estableció en el Plan Nacional de Desarrollo que el gobierno local "sea el principal artífice de su propio destino, con el apoyo del resto de la Federación, en este sentido, promoverá el desarrollo de planes concretos para cada región que sean acordes con las necesidades y vocaciones específicas, que sean congruentes con los procesos de descentralización económica, política y social que vive nuestro país”. A partir de estos compromisos, la Secretaría de Gobernación a través del Instituto Nacional para el Federalismo y Desarrollo Municipal (INAFED) elaboró el Programa especial para un Auténtico Federalismo.

Así pues Torres (2005) señala, que el Auténtico Federalismo se constituye como una forma de organización del gobierno ideal para otorgar coherencia a las políticas nacionales, promover la coordinación de las acciones de los tres ámbitos de gobierno, negociar y consensar con la diversidad para la definición de las directrices políticas básicas que requiere la nación y abrir espacios de consenso entre gobierno y sociedad.

Con el propósito de transitar hacia un Auténtico Federalismo, la Secretaría de Gobernación (2002) se plantea los siguientes objetivos:

- Impulsar la transferencia de facultades, funciones, responsabilidades y recursos de la Federación a los gobiernos locales de forma asimétrica y gradual, a fin de construir una coherente arquitectura de gobiernos con base en la descentralización política, la reestructuración territorial de la administración pública y la definición clara de las competencias gubernamentales. 
- Fortalecer a los gobiernos de las entidades federativas y de los municipios en sus capacidades de gestión pública, mediante un esquema integral de profesionalización y capacitación que apoye la descentralización, potencie la calidad de la administración, fomente la rendición de cuentas y abra cauces a la participación ciudadana.

- Impulsar relaciones intergubernamentales auténticamente federalistas con base en una adecuada infraestructura institucional y en la construcción de redes de políticas con orientación solidaria y subsidiaria.

- Coadyuvar en la promoción de un desarrollo regional equilibrado que impulse la competitividad, la inclusión social, la sustentabilidad, la promoción de innovaciones en el financiamiento de la infraestructura básica, el ordenamiento territorial y urbano; a fin de lograr la convergencia en las tasas de crecimiento, la mitigación de las disparidades regionales y la prosperidad conjunta de los miembros de la Unión.

- Fortalecer la transparencia y rendición de cuentas por medio de mejores mecanismos de control y fiscalización en el uso y destino de los recursos públicos, difusión y transparencia de la información pública, impulso a la investigación y constitución de acervos documentales federalistas, partiendo de la ampliación de los espacios de participación ciudadana en cada una de las fases que implican las políticas públicas.

Para cumplir con los anteriores objetivos se han establecido una serie de estrategias, cada una de las cuales contiene uno o más proyectos que se constituyen en sus líneas de acción. Uno de estos programas es Agenda para el Desarrollo Municipal (Descentralización Estratégica para el Desarrollo desde lo Local), del cual se profundizará más adelante.

La finalidad del Auténtico Federalismo es revertir la concentración de atribuciones y decisiones en el centro, afín de impulsar las potencialidades locales mediante una 
descentralización basada en la autonomía política de los estados, la renovación de la unidad nacional, la eficacia de la administración pública, el combate de las disparidades regionales y la revisión de los marcos institucionales a fin de fortalecer la república.

\section{Génesis de las RIG's.}

Como anteriormente se ha señalado, un elemento fundamental de federalismo es la distribución de poder entre los diferentes órdenes de gobierno que deben buscar la cooperación, colaboración y coordinación, buscando no anularse entre sí. Así el enfoque de las RIG's se convierte en una pieza fundamental para entender cómo se da la interacción entre los diferentes ámbitos de gobierno, para superar sus conflictos y mejorar la cooperación entre sí (González, 2006).

Uno de los principales argumentos para utilizar el concepto de RIG's es la existencia de problemas que requieren de la participación de los tres niveles de gobierno por lo que "la solución de problemas es un eje vital de las relaciones intergubernamentales" (Jaramillo, 2010).

Arturo Pontífes (2002) señala que al existir un acuerdo de relaciones pactadas, crean un orden normativo entre instituciones de gobierno; los convenios surgidos de este compromiso, permiten que las reglas programáticas, presupuestales y de control, tengan una mayor relevancia, por lo que la relación que exista entre los tres niveles de gobierno, se caracterizan por la suma de esfuerzos y la "concertación de acciones bajo una misma línea de trabajo".

Wright (1997) señala que el estudio de éstas comienza en los años treinta del siglo pasado en los Estados Unidos; un pionero de su estudio es William Anderson (Agranoff; 1997); quien 
las define como "un cuerpo importante de actividades o de interacciones que ocurren entre unidades gubernamentales ${ }^{5}$ de todos tipos y niveles dentro del sistema federal".

El enfoque de las RIG's trasciende los principios constitucional-legal, por lo cual, acerca tanto las divisiones entre gobiernos como sus respectivas funciones. Una de las ventajas de las RIG's radica en "no ignorar o infravalorar los análisis formales, sino que intentan superar los potenciales límites de la fuerte tradición jurídica” (Agranoff, 1997).

Aunque el concepto mismo se centra en las relaciones existentes entre los distintos niveles de gobierno y las instituciones que lo integran, son las relaciones personales, la tipificación de los problemas y la solución a éstos, las que enmarcan el desempeño y la interacción de las RIG's. De la agudeza de estos elementos depende en gran medida, el éxito o el fracaso en el diseño e implementación de las políticas públicas.

\section{Modelos de Aplicabilidad}

Las RIG's mantienen la idea de interacción entre diferentes centros de poder, por tal motivo, es conveniente especificar cuáles son los modelos de autoridad que presentan dichas relaciones.

Así pues, desde el punto de vista de Wright (1997) existen tres modelos los cuales a su vez muestran tres tipos de autoridad: la autoridad coordinada (autonomía), la autoridad dominante o inclusiva (jerarquía) y la autoridad legal o superpuesta (negociación), de tal forma que continuación se describen en forma más detallada en la siguiente gráfica.

\footnotetext{
${ }^{5}$ Las unidades de gobierno son definidas como "una entidad organizada que, además de tener carácter gubernamental, tiene suficiente discrecionalidad en la administración de sus propios asuntos para dirigirla por separada de la estructura separada de cualquier otra unidad de gobierno" véase (Wright, 1997: 73)
} 


\section{Diagrama 1. Modelos de las RIG's}

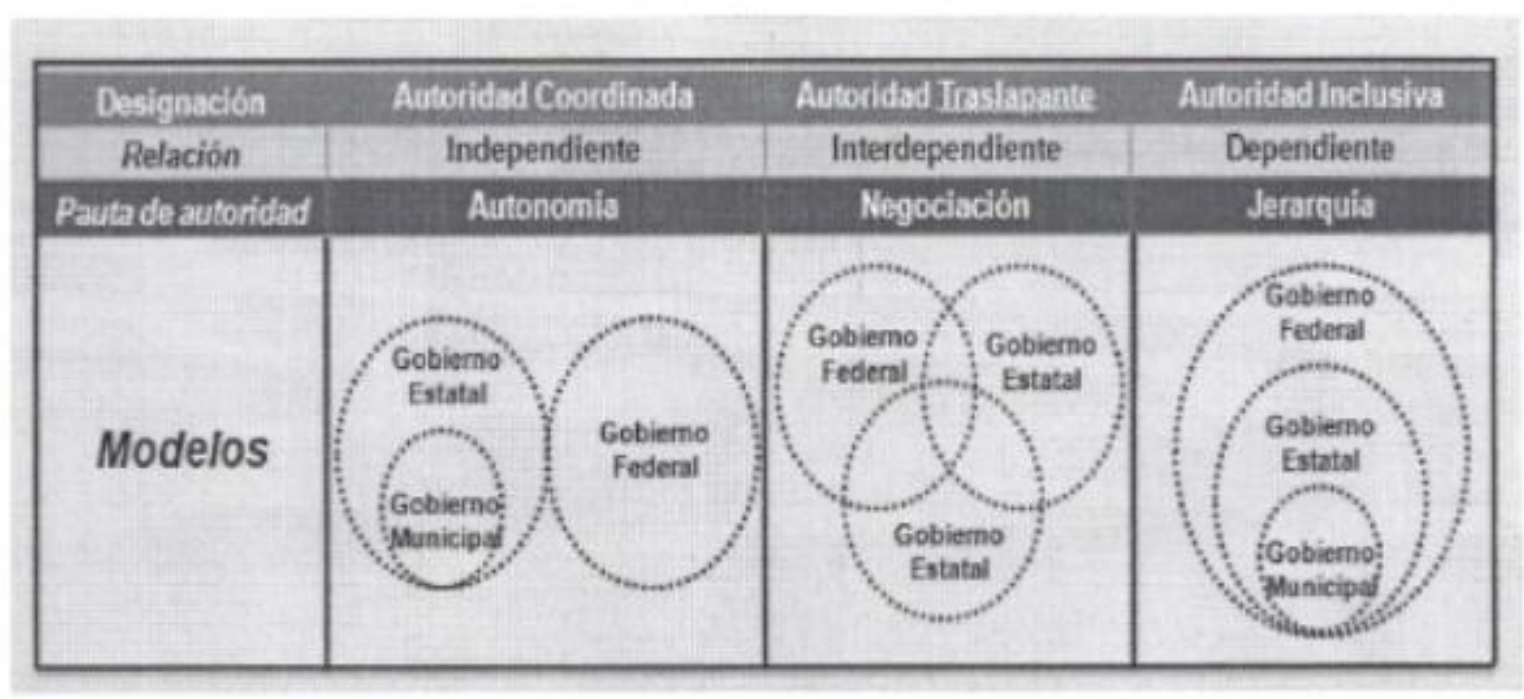

Fuente: Elaboración propia a partir de Wright, 1997.

Resumiendo los modelos de autoridad anterior, se podría decir que el modelo de autoridad coordinada plantea relaciones autónomas de autoridad nacional-estatal. En el modelo de autoridad inclusiva predomina la jerarquía y se considera a los gobiernos estatal y local como simples apéndices de un poderoso gobierno nacional que predomina el sistema centralizado. El modelo de autoridad superpuesta presenta a las RIG's como una conducta pautada interdependiente y negociada entre funcionarios nacionales, estatales y locales.

Se considera que el modelo que más se apega a lo que persigue el auténtico federalismo es el de autoridad superpuesta, ya que éste es el que establece las pautas para iniciar la negociación y la interdependencia entre las diferentes instancias de gobierno. 


\section{Políticas para Fortalecer a los Gobiernos Municipales}

El término políticas públicas hace referencia a los cursos de acciones y decisiones emprendidas por parte del gobierno y la sociedad para influir sobre un determinado problema de carácter público, pero también se utiliza como sinónimo de programas, metas, dicciones, normas, leyes e incluso de proyectos (Tamayo, 1997).

Al diseñar políticas destinadas al desarrollo local es importante la planificación que realicen los agentes locales que intervienen en el proceso, con el fin de aprovechar los recursos humanos y materiales que allí se encuentran. Además, la negociación y el diálogo entre los agentes económicos, sociales y políticos asentados en el territorio es fundamental para lograr ambientes propicios para las inversiones y mejorar el clima organizacional (Elizalde, 2003).

Aunque el tema de las políticas públicas tiene sus inicios en Estados Unidos (EEUU) con los trabajos pioneros de Haroll Lansswell en 1951 y 1971, en México este tema comenzó a recibir atención hasta años después que se planteó el problema de la reforma del estado a finales de la década de los ochenta.

El proceso de gestación de una política pública inicia en el momento en el que el gobierno identifica un problema en el que ve la necesidad de tomar decisiones para atenderlo. Por lo regular a partir del acopio de información se decide cómo atenderlo y darle solución; aunque también puede decidir no actuar. Como ya anteriormente ha mencionado, ambas acciones son una decisión de política pública. En la segunda etapa se produce la formulación de la política. Aquí es cuando se establece un plan o una serie de alternativas de la cual se elige la que se considera la más viable. Una vez que ya se eligió la opción a seguir, se inscribe en la agenda gubernamental y se continúa con su implementación. Este paso será el que mayor importancia tendrá en la presente investigación debido a que se trata de implementar el Programa Agenda para el Desarrollo Municipal. 
Mayntz y Schneider (citado por Subirats; 2008) señalan que la implementación como proceso de elaboración de las políticas públicas, envuelven a diferentes niveles gubernamentales, órganos administrativos y otros intereses afectados, constituyendo lo que se ha venido denominando redes de política pública (policynetwork o policycommunity), es decir, un entramado de actores institucionales políticos y sociales que hacen frente a una tarea o programa de actuación específica. La estructura de esos entramados y las interacciones entre sus actores tienen influencia en su rendimiento.

Finalmente, se debe destacar que las políticas públicas incluyen el ingrediente de la participación ciudadana y la acción de varios actores - entre ellos el gubernamental -el enfoque de las RIG's resulta de gran utilidad para comprender los distintos grados de efectividad que alcanzan las políticas, pues ayudan a tener en cuenta las características y dimensiones de los vínculos que se establecen entre unas y otras entidades gubernamentales, entre éstas y la sociedad.

Así, la triada Federalismo, RIG’s y políticas públicas, evidencia un marco de complementariedad deseable para mejorar la acción del gobierno: el federalismo tiene una de sus principales bases en las RIG's y éstas a su vez encuentran en las políticas públicas un importante apoyo para mejorar la coordinación entre entidades gubernamentales y entre actores participantes.

\section{El pilar para el Desarrollo del Sector Público}

La administración estratégica en las organizaciones públicas de acuerdo a Velasco (2010) para que tenga éxito debe seguir el siguiente modelo a) Generación de información sobre entorno externo e interno; b) Formulación de la estrategia (planeación estratégica); c) Implementación de la estrategia (ejecución de proyectos estratégicos); d) Evaluación de los logros; y e) Evaluación de la estrategia y del sistema de gestión estratégica. 
El enfoque de gestión estratégica ofrece importantes aportes a la labor de la función pública por el hecho que se enfoca principalmente a: a) Permite entender la misión del gobierno en términos de generación de un valor público superior para la sociedad; b) Reconocer que la generación de valor público depende de la capacidad de la organización por generar una ventaja competitiva o liderazgo; c) Establecer una alineación entre las condiciones del orden interno y los recursos o capacidades institucionales; y d) Exigir en la ejecución de las estrategias se debe contemplar el alineamiento de las actividades operativas y la visión institucional" (Velasco, 2010).

En este sentido, es necesario recordar que las organizaciones públicas existen para resolver problemas, satisfacer necesidades y generar oportunidades que son considerados de interés público. Hay que tomar en cuenta que el concepto de estrategia implica el entendimiento de la interactividad entre la organización y su entorno. Se debe partir de que la planeación estratégica tiene como propósito principal generar líneas de acción que resuelvan las necesidades, contemplando que existen diferentes formas en que se puede plantear una estrategia, la mejor de ellas es basados en la competencia; aquí se encuentra un problema puesto que a diferencia del sector privado, el sector público no cuenta con una competencia identificada, puesto que en el entorno mexicano el gobierno es el único ente capaz de brindar los servicios y por lo tanto, no cuenta con la misma competencia.

Porter (2010) explica que el estado de la competencia en una organización depende de cinco fuerzas básicas: la búsqueda por alcanzar una mejor posición entre competidores actuales la cual se ve acechada por amenaza de sus competidores, capacidad de negociación con los colaboradores, capacidad para negociar con los clientes y poder hacer frente a las amenaza de productos o servicios sustitutivos. Para ello, es necesario determinar cuál es la fuente de cada una de estas amenazas, y por lo tanto, poder establecer una estrategia capaz de dar solución a la problemática detectada. 
Las fuerzas competitivas determinan el grado de rentabilidad o éxito de una organización, y por tanto, son de gran importancia en la formulación de la estrategia. Una vez que se tiene el conocimiento pleno sobre el entorno, se pueden identificar las fortalezas y las debilidades de la organización, las cuales desde el punto de vista estratégico son el reflejo del estado que guarda la organización sobre las fuerzas que le afectan.

Bazaga (2010) señala que derivado de diversos acontecimientos políticos, económicos y sociales del mundo, las organizaciones públicas han optado por una progresiva especialización que las ha alejado de la visión global de su acción y ha disminuido su capacidad de influir en la totalidad del entorno, reformulando su manera de abordar los problemas. La acción pública se convierte en una relación de intercambio entre distintos actores. En suma, las organizaciones públicas desempeñan su actividad en contextos de gran turbulencia ambiental y son únicamente uno de los actores que están actuando en ellos, por lo tanto, han de enriquecer su información sobre la realidad y actualizarla constantemente.

Así que esta disciplina debe entenderse como un proceso mediante el cual se transforma el pensamiento, el conocimiento y el cambio organizativo. En conclusión la administración estratégica: a) debe producirse después de la reflexión y del pensamiento estratégico; b) en el marco de un proceso de gestión estratégica; c) el cual debe convertirse en un proceso dinámico.

Gobernar comienza a dejar de ser equivalente a previsión, mando y control universal, a decisiones desde lo alto, a controles verticales, y comienza a admitir la necesidad y dificultad de formular los propósitos claros y atractivos para su comunidad. Los gobernantes comienzan a admitir que existen factores limitantes externos y que es imprescindible sintonizarse con la dinámica del mundo exterior, coordinarse con ellos y hacerlos copartícipes y contemplar su fuerza. La administración estratégica es una tendencia a la planeación, dominante entonces en los sectores privados y públicos, ésta consiste básicamente en definir y realizar objetivos 
deseados, con singular interés por construir y consolidar una estrategia, que consiste básicamente en definir y alcanzar objetivos futuros (Aguilar, 2010).

\section{La AE en el Sector Público}

Antes de detallar el procedimiento que se recomienda seguir para estructurar adecuadamente un proceso de administración estratégica, es importante destacar los beneficios que traería éste para las organizaciones del sector público. Martínez y Ruíz (2007) argumentan que el proceso alienta la conciencia del cambio en la organización, ya que no basta solamente con enunciar intenciones de mejoras, sino de transitar a la acción y plantear objetivos alcanzables, proponiendo eficaces cursos de acción considerando las restricciones tanto en recursos humanos, como físicos, financieros y tecnológicos. Asimismo, traería solidez en el trabajo de la organización, ya que todos los miembros de ésta trabajarán en conjunto en la búsqueda de objetivos comunes aplicando a su vez estrategias también comunes.

Seguir un proceso de AE permite a la organización elevar su capacidad para relacionarse con otros actores y contemplar que el éxito de una estrategia no es una cuestión exclusiva de los tomadores de decisiones y que tampoco depende de su formulación, sino de la manera en la que intervienen otras instancias en su implementación (Davis, 2008).

Dado que la AE propicia el trabajo conjunto entre las unidades de la organización, en éstas debe existir una comunicación constante y fluida sin restricciones que dé como resultado que la interacción de agentes no se encuentre limitado por su nivel jerárquico, ya que esto es lo que hace funcionar a los participantes como un equipo competitivo.

Otra de las bondades que se localizan en este proceso, es que se asegura que una organización formule estrategias apropiadas que le permita cubrir sus necesidades en un momento determinado y limite los factores que amenazan su futuro (Martínez y Ruíz, 2007). 
Por otra parte, representa un proceso sistemático, lógico y objetivo para tomar decisiones importantes en una organización, ya que en éste se obtiene y ordena información cualitativa y cuantitativa de tal manera que propicie la toma de decisiones eficientes muy a pesar de la incertidumbre que muestre el ambiente que rodea a la organización (Davis, 2008).

Este proceso ayuda a identificar las amenazas externas, permitiendo una mayor comprensión de las estrategias de los competidores, incrementa la productividad de los empleados, dado que es un proceso dinámico que minimiza la oposición al cambio, refuerza la capacidad de las organizaciones para prevenir problemas porque fomenta una mayor interacción de los integrantes de la organización no importando su nivel jerárquico (Dess, 2011).

Para Gallardo (2012) la AE es un proceso que muestra una serie de beneficios que vale la pena exaltar porque: a) unifican los criterios de hacia dónde se dirige la organización; b) identifica las principales variables de la organización que le permita lograr el éxito; c) mejora la visión a largo plazo; d) revela y controla las amenazas futuras; e) identifica los asuntos estratégicos y f) genera un encadenamiento entre objetivos y medios para alcanzarlos.

Finalmente, permite que la organización, en este caso el municipio, tome parte activa en la configuración de su futuro, ya que puede emprender actividades e influir en ellas y por consiguiente controlar su destino; en este proceso resulta vital el diálogo e involucramiento entre los participantes quienes se vuelven parte importante para alcanzar los éxitos; la participación es clave para conseguir los compromisos con los cambios que se requieren (Davis, 2008).

\section{Método a Seguir para Aplicar un Proceso de AE}

La literatura especializada en el tema de la AE muestra que para la iniciativa privada existen una serie de elementos indispensables que se deben de contemplar y seguir para desarrollar este proceso. Cabe resaltar que en las organizaciones públicas, no existe en general un 
modelo a seguir, sin embargo, el esquema metodológico que en la presente investigación se muestra tiene semejanza con la elaboración de políticas públicas, anteriormente señalado, ya que en éste, una vez que se detecta un problema de carácter público, se formulan alternativas de solución; se desarrolla un esquema racional de puesta en práctica donde se identifican los insumos necesarios para este fin y posteriormente se evalúa el impacto de las acciones y el logro de objetivos. En el siguiente diagrama de la gráfica 2, se desarrolla un modelo básico de AE con las preguntas que intentan responder cada una de las fases:

\section{Diagrama 2. Proceso de Administración Estratégica}

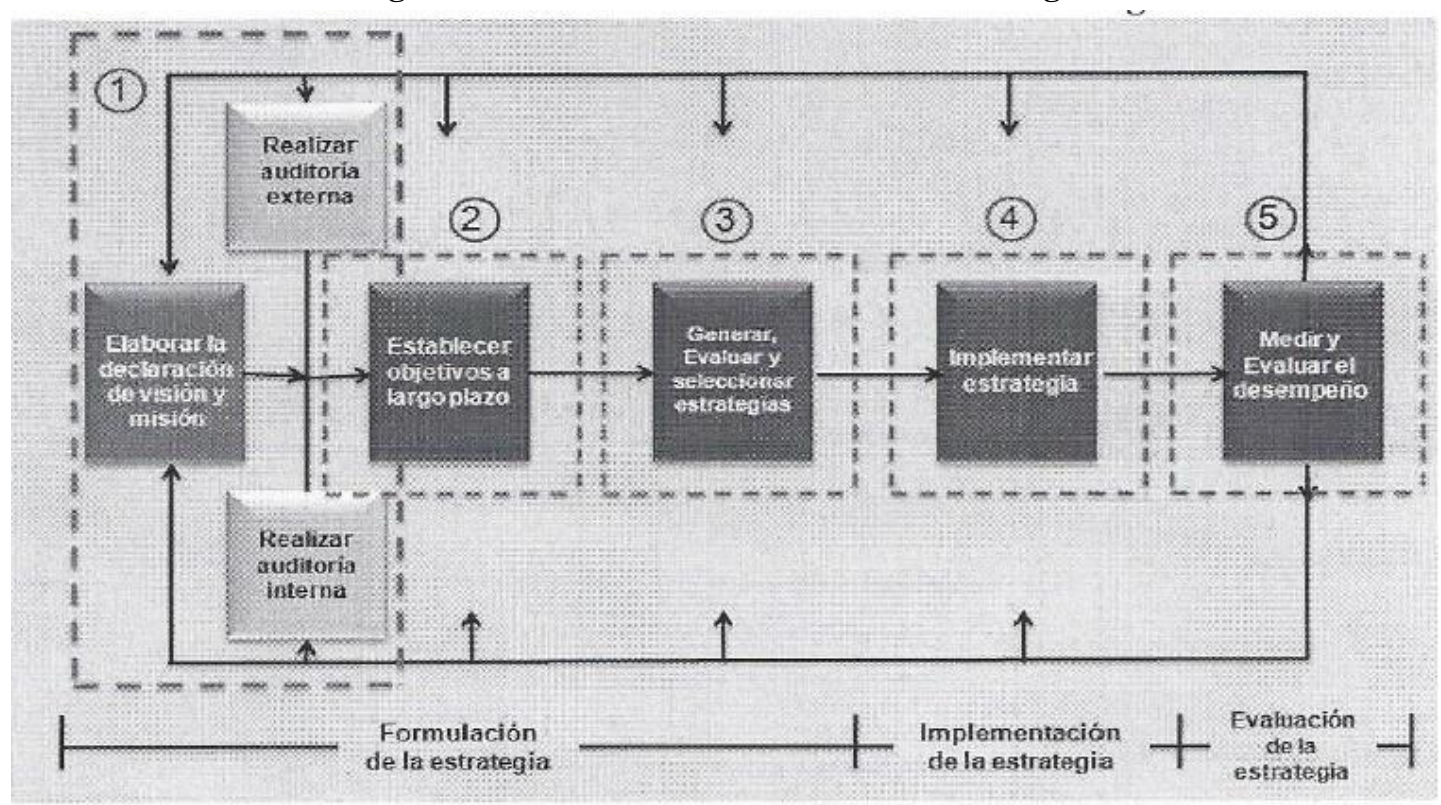

Fuente: Elaboración con base en Davis (2008), Armijo (2011) y Dress (2012)

En la primera fase del proceso se genera un análisis de las metas trascendentales de la organización (visión, misión y objetivos estratégicos) junto con un análisis sistemático de su contexto endógeno y exógeno que permite identificar las fortalezas, debilidades, oportunidades y amenazas; partiendo de ello para construir una serie de alternativas o estrategias que habrán de seguirse para alcanzar los objetivos, potenciar las fortalezas y oportunidades y mitigar debilidades y amenazas. Se debe contemplar que las alternativas 
formuladas deben crear ventajas competitivas para la organización Serna (2000), Davis (2008) y Dess (2011).

Como se ha indicado anteriormente, el punto de partida es establecer la misión y la visión de la organización; esta tarea le pertenece a los encargados de tomar decisiones directivas, es decir, compete a los más altos niveles de responsabilidad organizacional. En la misión se definen los productos finales o estratégicos y los resultados por los cuales la organización debe responder a sus usuarios (Armijo, 2011).

Davis (2008) señala que la misión representa una declaración del propósito que mueve a la organización y las distingue de otras similares, es decir, en ésta se expresa la razón de ser de la organización y resulta fundamental por el hecho de que una declaración clara de la misión resulta esencial para establecer objetivos y formular estrategias de manera eficaz.

La declaración de la misión pone de manifiesto lo que la organización quiere ser y a quién sirve; tiene que ver con las siguientes preguntas: ¿Quiénes somos, qué hacemos, para quiénes y qué efectos o resultados finales esperamos? El establecimiento de la visión debe ser realista y creíble, bien articulado y fácilmente entendible. Debe orientar la energía de la organización, servir como guía y ser congruente con sus valores. En términos de Arellano (2004), "la visión debe significar un reto y a la vez una inspiración para el logro de su misión".

En este sentido la misión del gobierno municipal de Mexicali es "definir las acciones y estrategias que impulsen una administración municipal eficiente, que maximice los recursos y reoriente el significado del servicio público con una visión participativa y transparente; que enlace acciones concretas dentro del periodo legal de la administración municipal con una perspectiva a largo plazo; que defina las aspiraciones de una sociedad en plena evolución; que finque acciones hoy, que apuntalen otras de mayor contundencia en su impacto futuro" (XX Ayuntamiento de Mexicali, 2011).

Por otra parte, Thompson (2012) señala que la visión corresponde al futuro deseado de la organización. Se refiere a cómo quiere ser reconocida la entidad y representa los valores con 
los cuales se fundamentará su accionar público y responde a la pregunta: ¿Cómo queremos ser reconocidos?

La visión es la perspectiva que los miembros de una organización buscan hacer, es una especie de imagen ideal racional sobre el quehacer de la organización (Arellano, 2004). En las organizaciones públicas, la visión enmarca las aspiraciones institucionales, permite distinguir y visualizar el carácter público, ya que detalla lo que la organización entrega a la sociedad y muestra los beneficios que la sociedad espera de la entidad pública (Armijo, 2011).

\section{Descentralización Estratégica para el Desarrollo Desde Lo Local}

Un buen ejemplo del proceso que anteriormente hemos descrito lo representa el programa Agenda para el Desarrollo Municipal el cual se desprende del programa macro denominado Hacia un Auténtico Federalismo, y especifica una serie de estrategias para impulsar el desarrollo de los gobiernos municipales. (INAFED, 2012).

Agenda para el Desarrollo Municipal es un programa y una metodología que se traduce en términos de medición, en una descentralización estratégica que se enfoca a que todos los municipios participantes en el programa tengan las condiciones mínimas en las que tiene que operar su gestión municipal.

El INAFED enmarca una serie de beneficios que logran obtener los municipios participantes de este proyecto entre los cuales se encuentran los siguientes:

- Cuentan con una herramienta que les permite conocer el estado que guarda su administración.

- Programan acciones específicas para la superación de rezagos localizados en la etapa del autodiagnóstico.

- Priorizan la aplicación de sus recursos de acuerdo con las necesidades detectadas.

- Permite generar una evaluación sobre el desempeño de sus funciones y empleados.

- Reciben una certificación sobre las prácticas favorables. 
Este programa, se desarrolla a través de 39 indicadores, los cuales como anteriormente se ha señalado, permiten conocer las fortalezas y debilidades de la gestión municipal; estos indicadores están distribuidos en las siguientes categorías: 1) Desarrollo Institucional para un buen Gobierno; 2) Desarrollo Económico Sostenible; 3) Desarrollo Social Incluyente; y 4) Desarrollo Ambiental Sustentable.

El INAFED específica que cada uno de los indicadores y parámetros de medición que están plasmados en el cuadernillo del autodiagnóstico, cuentan con tres niveles de medición que se asemejan a los de un semáforo, en los cuales se estipulan la situación en la que se encuentra el municipio.

\section{Modelo de Gestión para el fortalecimiento Municipal}

Construir un modelo de gestión como el que aquí se muestra en la gráfica 3 es de relevancia ya que implicaría tomar en cuenta los elementos anteriormente citados, en este sentido un modelos de debe estar estructurado de forma tal que las organizaciones dividan sus labores en distintas actividades para después coordinarlas (Santos, 2014). En este sentido, la búsqueda por el desarrollo de los procesos organizacionales y de nuevas perspectivas que garanticen una buena gestión, han llevado a plantear estrategias o modelos de gestión que intenten asegurar un fortalecimiento en la administración local.

Por tal motivo el modelo debe partir de la idea que para fortalecer a las instancia gubernamentales a nivel local debería darse un proceso de interacción en el que una diversidad de actores en el ámbito de sus competencias, generen acciones y estrategias encaminadas a resolver los problemas comunes; para que esta interacción se desarrolle adecuadamente, se requiere que exista una constante comunicación e intercambio de información entre los participantes, así como el desarrollo de una conducta favorable que propicie que la comunicación e información fluyan adecuadamente (Guerrero, 2001). El segundo elemento que debe contemplar un modelo de gestión, es que se formulen verdaderas políticas públicas a partir de las prioridades localizadas por los gobiernos locales y dejar atrás 
el viejo esquema incrementalista, en donde las estrategias a seguir eran formuladas e implementadas por órdenes de gobierno ajenos al entorno local.

Para formular e implementar las políticas locales, es indispensable generar un proceso de administración estratégica en donde inicialmente se desarrolle un diagnóstico que sea capaz de localizar tanto las fortalezas como las debilidades con las que cuenta actualmente la administración municipal; esta etapa en el modelo se asemeja a lo propuesto por Agenda para el Desarrollo Municipal, el cual persigue ese propósito, a través de un auto diagnóstico.

Finalmente, hay que destacar la necesidad que existe de contar con una adecuada interacción entre los diferentes actores involucrados en el proyecto y que de estos puedan generarse redes de políticas que coadyuven a una adecuada implementación de las estrategias encaminadas a resolver problemas públicos. En estas redes de políticas se debe destacar la figura de un Estratega que tenga la capacidad política de poner en concordancia, adecuadas relaciones intergubernamentales ya que Agenda para el Desarrollo Municipal pretende ser un pilar innovador para la generación de un modelo de gestión como el ya descrito.

Diagrama 3. Modelo de Gestión para el Fortalecimiento de la Administración Municipal

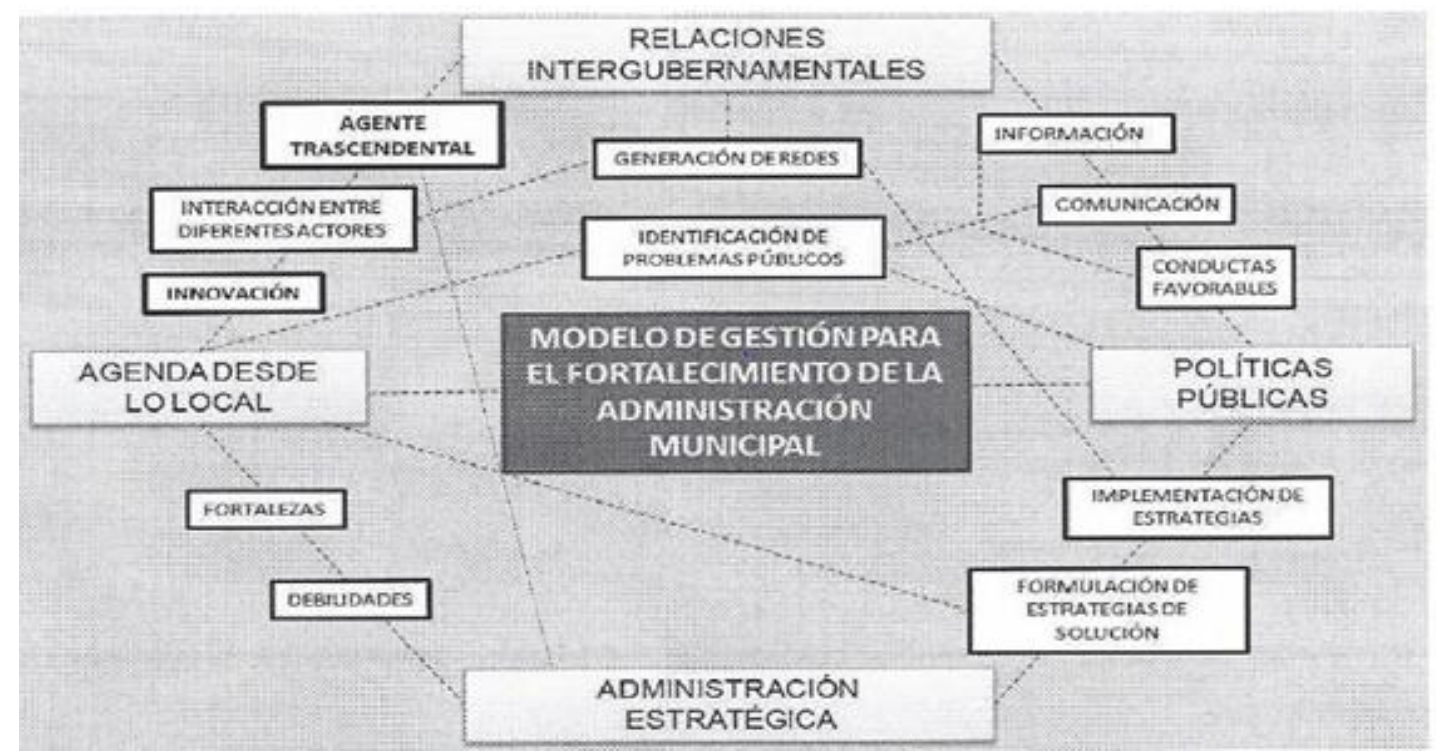

Fuente: Elaboración propia con base en Wrigth (1997), Guerrero (2001) y Cabrero (2008). 


\section{Conclusiones}

Fortalecer al gobierno municipal a través de un Modelo de Gestión como el que aquí se propone, implica un proceso de modernización constante, donde se ponga en juego la capacidad de este orden de gobierno para fomentar y consolidar verdaderas Relaciones Intergubernamentales que repercutan en la construcción de redes de políticas. Asimismo, implica formular políticas públicas con una visión racional y bajo las necesidades del orden local; además se debe consolidar un proceso de administración estratégica donde se tenga vasto conocimiento de las cuestiones endógenas y exógenas que pudiesen afectar a la administración municipal, partiendo de ahí hacia la construcción de estrategias con visión de largo plazo.

El modelo de Gestión que resultaría indispensable poner en funcionamiento para fortalecer la administración municipal, tiene como principal elemento la puesta en marcha de un proceso de administración estratégica aplicado al ámbito local, por lo cual consideramos que el programa Agenda para el Desarrollo Municipal, representa un proyecto ambicioso y cubre los requerimientos antes señalados para cambiar la administración por la gestión, para que en el mediano plazo se tengan repercusiones positivas en el municipio.

El aprendizaje que ha arrojado el análisis del presente proyecto de investigación, es que por medio del programa Agenda para el Desarrollo Municipal, se genera un proceso de lineamientos entre los propósitos del municipio y los focos rojos localizados en el autodiagnóstico. Esto permite que exista una coherencia en la implementación de acciones y resulta primordial para conocer el rumbo que debe tomar la administración municipal, lo cual es sin duda una de las mayores virtudes del programa.

Resulta fundamental para fortalecer al ámbito local, contar con herramientas como el programa Agenda para el Desarrollo Municipal, ya que éste no sólo se enfoca en la mejora de la prestación de servicios, sino a la implementación de una serie de programas federales que buscan apoyar a los gobiernos municipales y así, aproximarlos a instrumentos de gestión específicos para su buen funcionamiento. 


\section{Referencias}

A Aguilar, Villanueva, L.F., “Gobernanza y Gestión Pública”, México: Fondo de Cultura Económica. (2006),

Agranoff, R. "Las relaciones y la Gestión Intergubernamental”, en: Bañón R. y Carrillo E. (comp.), La nueva Administración Pública, Madrid: Alianza Universidad. (1997)

Armijo, M., "Planificación estratégica e indicadores de desempeño en el sector público", (2011) CEPAL - Serie Manuales $\mathrm{N}^{\circ}$ 69, extraído de: http://www.eclac.cl/cgibin/getProd.asp?xml=/publicaciones/xml/8/44008/P44008.x $\mathrm{ml} \& \mathrm{xsl}=/ \mathrm{ilpes} / \mathrm{tpl} / \mathrm{p} 9 \mathrm{f} . \mathrm{xsl}$

Cabrero, E., "Políticas Públicas Municipales una agenda en construcción”, México, Porrúa. (2003)

, “Capacidades institucionales en gobiernos subnacionales de México”. ¿Un obstáculo para la descentralización fiscal? Revista Gestión y Política Pública. XIII, (3) (2004)

Recuperado

de http://www.gestionypoliticapublica.cide.edu/num_anteriores/Vol.XIII_NoIII_2dose $\mathrm{m} /$ Cabrero.pdf

, "Innovación local en América Latina", Centro de Investigación y Docencia Económicas (CIDE), Liaison Group, Observatorio Latinoamericano de la Innovación Pública Local, México, p. 332. (2008).

Davis, F., "Conceptos de administración estratégica", 11ª Ed., México: Pearson Prentice Hall. (2008).

Dess, G., Lumpkin, G. y Eisner, A., "Administración estratégica textos y casos”, (5a. ed.). México: McGraw-Hill. (2011)

Elizalde, A., "Planificación estratégica territorial y políticas públicas para el desarrollo local”. (2003) CEPAL serie gestión pública No.29 http://www.eclac.org/publicaciones/xml/2/11852/sgp29.pdf

Gallardo, J.R. “Administración estratégica de la visión a la ejecución”. México: Alfaomega. (2012)

González, J.C., "Programa nacional de becas para estudios superiores. Un enfoque de relaciones intergubernamentales", en espacios públicos, febrero, año/vol.9 número 017 UAEM, Toluca pp $276 \quad 291, \quad$ (2006). http://redalyc.uaemex.mx/pdf/676/67601716.pdf

Goodstein, L. y otros "Planeación Estratégica Aplicada", Colombia: MCGRAW-HILL. (2001),

Guerrero O., "Nuevos Modelos de Gestión Pública” Revista Digital Universitaria, Vol. 2 , (2001) Num.3 Extraído de: http://98.131.112.222/docs/Lecturas/Nuevos_modelos_de_gestion_publica.pdf

Guillen T. "Federalismo, Gobiernos Locales y Democracia", IFE, Cuadernos de la cultura democrática No. 17 México (2001). 
Guzmán J., "El federalismo en la modernización y fortalecimiento de los gobiernos municipales", México, Instituto de Administración Pública del Estado de Querétaro, A.C. (1995).

Instituto Nacional para el Federalismo y Desarrollo Municipal (2012), Documentos de trabajo de Agenda para el Desarrollo Municipal, recuperado de: http://www.inafed.gob.mx/es/inafed/inafed_adll_documentos_trabajo

Jaramillo C. "La descentralización: una mirada desde las políticas públicas y las relaciones intergubernamentales en Baja California" en Región y sociedad vol.22 no.49, (2010). Recuperado de: http://www.scielo.org.mx/scielo.php?script=sci_arttext\&pid=S18703925201000030 0007

Martínez, O.C y Ruiz J.G "Aproximación a la situación estratégica del sector restaurantero en la ciudad de Tijuana, Baja California, México", en Administración Estratégica. México: Printed in México. (2007).

Mejía Lira, J. La importancia de las redes inter e intragubernamentales, en Gaceta de Administración Pública Estatal y Municipal, no. 60. INAP, México. (2003).

Méndez, J., Becerril, T. "Planeación estratégica: técnica gerencial aplicada en la administración pública”, en Quivera, vol. 7, núm. 2, julio diciembre, (2005) extraído de: http://www.redalyc.org/articulo.oa?id=40170207

Moreno Toscano, A. "Descentralización y Federalismo", Instituto de Estudios Políticos, Económicos y Sociales, México, (1998).

Navas Quintero, A. "La nueva gestión pública: una herramienta para el cambio", en Perspectiva, número 23, pp. 36-38. (2010) extraído de: http://www.revistaperspectiva.com/archivos/revista/No\%2023/036038\%20PERS\%2 0OK.pdf

Peters, G. "Gobernanza y burocracia política: ¿nuevas formas de democracia o nuevas formas de control?", en Foro Internacional." Vol. XLV, número 4. (2005) Extraído de:http://codex.colmex.mx:8991/exlibris/aleph/a18_1/apache_media/AK3S9HN8M 53Y1X3U77KM4DE33Q7KL5.pdf

Porter, M., “¿En qué consiste la estrategia?”, en: Harvard Business Review NovemberDecember. Traducido en versión libre por la Fundación para el Desarrollo Integral del Valle del Cauca FDI y el Centro de Productividad del Pacífico. (1996) Extraído de: http://data7.blog.de/media/740/6344740_cb9423dd71_d.pdf

Sánchez, A, y García, M. "Las capacidades de los gobiernos municipales para instrumentar política social”. (2007). Recuperado de http://www.urbared.ungs.edu.ar/textos/IGLOM/Mesa4_doc3.pdf

Santos, L. "Modelos de gestión: hacia un nuevo derecho procesal organizacional" Revista de Derecho Público, Colombia. (2014) Recuperado en: http://web.a.ebscohost.com/ehost/pdfviewer/pdfviewer?vid=7\&sid=8851dd3c-f89043f5-a7fe-d5b9c11 fce8f\%40sessionmgr4001\&hid=4112 
Secretaría de Gobernación, "Programa especial para un Auténtico federalismo". (2002)

Subirasts, J., Knoepfel, P., Laurre, C., \&Varone, F. "Análisis y Gestión de Políticas Públicas". Barcelona: Ariel. (2008).

Tamayo. "El análisis de las políticas públicas", en R. Bañón y E. Carrillo (comp.), La nueva Administración Pública, Madrid Alianza Universidad. (1997).

Torres, C, "La coordinación gubernamental: mitos y realidades del sistema federal mexicano", en X Congreso Internacional del CLAD sobre la Reforma del Estado y de la Administración Pública, (2005) Recuperado de: http://cdim.esap.edu.co/BancoMedios/Documentos\%20PDF/la\%20coordinaci\%C3 $\%$ B3n\%20gubernamental.pdf

Uvalle, Berrones R. "Federalismo y Gobernabilidad y políticas públicas en revista" IAPEM no. 50, México. (2001)

Vásquez-Barquero, A. "Desarrollo endógeno y globalización". EURE Revista Latinoamericana de Estudios Urbano Regionales, 26, 79, (2000). Recuperado de: http://www.scielo.cl/scielo.php?pid=S0250-71612000007900003

Wright, Deil S. "Para entender las relaciones intergubernamentales", México: Fondo de Cultura Económica. (1997);

Ziccardi, Alicia "Políticas sociales y gobiernos locales en el federalismo". (2002) Recuperado http://www.congresoretosyexpectativas.udg.mx/Congreso\%203/Mesa\%202/Mesa2_ 24.pdf 